\title{
Revista de las Indias (1938-1950): La difusión cultural y el mundo letrado ${ }^{1}$
}

\section{Resumen}

En el mes de julio de 1936 apareció el primer número de la Revista de las Indias. A partir de 1938, la política editorial de la Revista apostó por una mirada más allá de las fronteras nacionales que trató de abarcar el continente temáticamente. Este cambio en el enfoque se instaló en una coyuntura compleja: la Guerra Civil Española (19361939), la Segunda Guerra Mundial (1939-1945) y el cambio de gobierno de Alfonso López Pumarejo a Eduardo Santos (1938-1942). El contexto llevó a la Revista a poner énfasis en temas novedosos como difundir y comprender el quehacer letrado colombiano y el que se producía en lengua española, lo que permitió prestar atención a la situación de este tipo de trabajos en un momento de crisis; por eso, entre septiembre de 1944 y abril de 1945, la Revista elaboró y publicó las respuestas a cuatro preguntas de hombres de letras nacionales y extranjeros sobre las consecuencias que tendría la guerra para el mundo letrado en América. El presente texto propone una descripción de este proyecto editorial y cómo se percibía el devenir del quehacer intelectual en un momento de crisis internacional.

Palabras clave: Colombia siglo XX, historia intelectual, historia cultural.

Referencia para citar este artículo: BETANCOURT MENDIETA, Alexander (2016). "Revista de las Indias (1938-1950): La difusión cultural y el mundo letrado". En Anuario de Historia Regional y de las Fronteras. 21 (2). pp. 125-147.

Fecha de recepción: 06/11/2015

Fecha de aceptación: 16/02/2016

\begin{abstract}
Alexander Betancourt Mendieta: Doctor en Estudios Latinoamericanos, Universidad Nacional Autónoma de México. Miembro correspondiente de la Academia Colombiana de Historia. Profesor investigador de la Facultad de Ciencias Sociales y Humanidades, Universidad Autónoma de San Luis Potosí, México. Correo electrónico: alekosbe@uaslp.mx.
\end{abstract}

\footnotetext{
1 Trabajo contemplado en el proyecto Latinoamericanismo, Panamericanismo y Conmemoraciones: estudios comparados en América Latina, 1940-1970 (CONACyT CB 169284). Una versión preliminar se presentó en el XVII Congreso Colombiano de Historia, octubre del 2015. Se agradece el apoyo recibido por Paola Viviana Londoño de la Biblioteca Nacional de Colombia y Pedro Pablo Hernández Ortega de la Biblioteca Luis Ángel Arango como apoyo para consultar y ubicar algunos materiales usados en la realización de este trabajo.
} 


\title{
Revista de las Indias (1938-1950): Cultural Dissemination and the Literate World
}

\begin{abstract}
In July 1936, the first issue of the Journal of the Indies was published. From 1938, the editorial policy of the Magazine opted for a view beyond national borders which thematically tried to cover the continent. This shift in focus was installed amid a complex situation: the Spanish Civil War (1936-1939), World War II (1939-1945) and the change of government from Alfonso Lopez Pumarejo to Eduardo Santos (1938-1942). The context led the magazine to emphasize new issues to disseminate and understand Colombian literate work and the one produced in Spanish, which allowed drawing attention to the situation of this type of work in times of crisis. That is why, between September 1944 and April 1945, the magazine produced and published the answers to four questions made by literate Colombians and foreigners on the consequences of war for the literate world in America. This paper proposes a description of this editorial project and how the evolution of intellectual work was perceived in a time of international crisis.
\end{abstract}

Keywords: Colombia Twentieth Century, intellectual History, Cultural History.

\section{Revista de las Indias (1938-1950): A difusão cultural e o mundo letrado}

\section{Resumo}

No mês de julho de 1936 surgiu o primeiro número da Revista de las Indias. A partir de 1938, a politica editorial da Revista apostou numa abordagem além das fronteiras nacionais, que tentou abarcar tematicamente o continente. Esta mudança no enfoque foi instalada numa conjuntura complexa: a Guerra Civil Espanhola (1936-1939), a Segunda Guerra Mundial (1939-1945) e a troca de governo de Alfonso López Pumarejo a Eduardo Santos (1938-1942). O contexto levou à Revista a enfatizar nos temas de novidade para difundir e compreender o trabalho letrado colombiano e o que era produzido na língua espanhola, o qual permitiu prestar atenção à situação deste tipo de trabalhos em um momento de crise. Por isto, entre setembro de 1944 e abril de 1945 a Revista realizou e publicou as respostas a quatro perguntas de homens de letras nacionais e estrangeiros sobre as consequências que teria a guerra para o mundo letrado na América. O presente texto propõe uma descrição deste projeto editorial e como era percebido o futuro do trabalho intelectual num momento de crise internacional.

Palavras chave: Colombia século XX, história intelectual, história cultural. 


\title{
La Revista de las Indias: ¿un medio cultural del partido liberal?
}

La aparición de la Revista de las Indias fue una decisión administrativa del Ministerio Nacional de Educación del gobierno colombiano. La medida buscaba centralizar en una sola dependencia el esfuerzo coordinado, sistematizado, de los diversos órganos de divulgación cultural. La Revista sustituyó a Senderos que hasta ese momento había sido el medio de difusión oficial de la Biblioteca Nacional de Colombia. La nueva publicación era parte de la política de "[...] extensión cultural como órgano del Ministerio de Educación, [que] obedece al plan meditado de un programa de difusión [...] para mantener un contacto directo entre el Ministerio y la ciudadanía"2 que justificaba su decisión a partir de la siguiente pretensión:

\begin{abstract}
Hoy se quiere hacer de REVISTA DE LAS INDIAS una cátedra de alta cultura, dando cabida en sus páginas a estudios de toda índole [...] esa labor es ante todo de una urgencia inmediata para mantener una tónica elevada en las disciplinas de la inteligencia ${ }^{3}$.
\end{abstract}

Hay tres aspectos para resaltar en este trabajo sobre la Revista de las Indias. El de revisar cómo la publicación se inscribe en una política institucional del gobierno colombiano relacionada con la difusión de la cultura y cómo es matizada con una nueva perspectiva que se plantea claramente en la justificación y patrocina a la Revista de las Indias como una revista de alta cultura. El otro asunto que se quiere resaltar es que gracias a la situación de cambios generalizados que estaba viviendo Colombia en el momento de aparecer la publicación, se abrió la posibilidad de discutir y analizar el Estado social del país y la necesidad de que el gobierno central atendiera el problema de la construcción de la nación colombiana a través de acciones concretas para enfrentar el presente y la planeación del futuro en un escenario incierto a nivel interno y externo. Por eso, el tercer asunto a tratar es la situación de la cultura letrada y el papel de los hombres de letras en la sociedad y en el futuro a partir de los indicios que da el ejercicio de la encuesta que elaboró y difundió la revista.

La Revista de las Indias es un objeto referido de manera reiterada en los trabajos que estudian el periodo de la República Liberal (1930-1946) en la historia de Colombia; sin embargo, la referencia a dicha revista no implica ahondar en su desenvolvimiento ni en sus alcances específicos. Un lugar común en esta dirección privilegia la perspectiva política para explicar todos los procesos históricos pero las limitaciones de un enfoque obtuso de la política y su rol en la sociedad justifica, de manera simplista, la afirmación que explica todo el devenir de la Revista de las Indias con observar un hecho obvio: es una publicación oficial porque era dependiente de un ministerio del gobierno liberal de aquel momento; incluso se llega a afirmar que el propósito de ensanchar el pensamiento colombiano a través de las ideas culturales del " [...] proyecto liberal no logró cristalizar completamente sus propósitos debido a las contradicciones internas

\footnotetext{
${ }^{2}$ Revista de las Indias, vol. I, núm. 1, Bogotá, Ministerio de Educación Nacional, 1936, p. 1.

${ }^{3}$ Ibid.
} 
del partido y la diferencia de pensamiento de algunos de sus corresponsales" ${ }^{4}$. Las caracterizaciones de este tipo impiden avanzar en el análisis detallado de algunas situaciones generales que supone la formulación e implementación de la política cultural que se dio en el periodo de la República Liberal y de la Revista de las Indias como producto intelectual concreto de esas iniciativas, así como de sus alcances.

El reconocimiento del valor de la aproximación de la historia política, sin caer en la simplificación partidista, para explicar un fenómeno cultural como la Revista de las Indias se encuentra en el marco histórico en el que se hizo la aparición de esta publicación, caracterizado fundamentalmente con las preocupaciones acerca del presente y el futuro del Estado nacional. En este periodo, un tema central era tomar una posición sobre los roles del Estado en la sociedad a partir de las medidas que habían efectuado los gobiernos fascistas en Europa en materia educativa y cultural. Las aproximaciones a estas preocupaciones en Colombia se pueden encontrar en los debates sobre las reformas educativas que se implementaron en las décadas de 1930 y 1940. Estas tomas de posición se dieron en el preciso momento en que el ejercicio del gobierno nacional recayó en manos del partido liberal, al mismo tiempo que muchos sectores de la opinión pública colombiana, no solo los integrantes del partido conservador, reconocían que Alemania y sus aliados ganarían la guerra e impulsarían un nuevo orden mundial, lo cual, en términos prácticos, implicaba la desaparición de la democracia liberal como modelo para imponer el orden y el progreso ${ }^{5}$.

La forma que adquirieron los debates parlamentarios y los textos de opinión publicados en los periódicos y las revistas no se detuvieron sobre la factibilidad técnica y económica, o la utilidad para el futuro del país, sino que tuvieron iniciativas como la creación y las reformas de instituciones como la Universidad Nacional y la Escuela Normal Superior ${ }^{6}$; más bien, las discusiones hicieron énfasis en los intereses coyunturales a través de argumentos que aspiraban a movilizar cierto fervor nacionalista marcado en hitos como: la cultura hispánica, la herencia católica, la reinterpretación de las propuestas de Simón Bolívar sobre la organización del Estado y la utilidad de

\footnotetext{
${ }^{4}$ Acevedo Gaviria, Claudia Patricia. Intelectuales, criticos y modernidad cultural. Los casos de Baldomero Sanín Cano, Hernando Téllez y Jorge Zalamea, (tesis doctorado), UNC, 2013, p. 32; Martínez Hernández, Lina María. La Revista de las Indias (1936-1938): sus intelectuales como pensadores y ejecutores de la reforma educativa y cultural (Bogotá: Universidad de los Andes, 2011).

${ }^{5}$ Sierra Mejía, Rubén. "Política y cultura durante la República Liberal”, en Sierra Mejía, Rubén (ed.), República Liberal: sociedad y cultura (Bogotá: Universidad Nacional de Colombia, 2009), pp. 360-367; Ayala Diago, César Augusto. El porvenir del pasado. Gilberto Álzate Avendaño: sensibilidad leoparda y democracia. La derecha colombiana de los años treinta (Bogotá: Fundación Gilberto Álzate AvendañoUniversidad Nacional de Colombia, 2007); Ayala Diago, César Augusto. Inventando al Mariscal: Gilberto Álzate Avendaño: circularidad ideológica y mímesis política (Bogotá: Fundación Gilberto Álzate Avendaño-Universidad Nacional de Colombia, 2010).

${ }^{6}$ Ministerio de Educación Nacional. La obra educativa del Gobierno en 1940: la extensión cultural en Colombia, t. 3 (Bogotá: Imprenta Nacional, 1940); Tirado Mejía, Álvaro. Aspectos políticos del primer gobierno de Alfonso López Pumarejo: 1934-1938 (Bogotá: Instituto Colombiano de Cultura, 1981); Morales Benítez, Otto (comp.). Baldomero Sanín Cano: ideología y cultura (Bogotá: Universidad Externado de Colombia, 1998).
} 
tener gobiernos autoritarios y paternalistas ${ }^{7}$; por eso, los argumentos en contra de las iniciativas gubernamentales en materia de educación y cultura se concentraron en los señalamientos sobre el abandono de la tradición histórica del país -el catolicismoy la impugnación sobre la implementación de ideologías foráneas revestidas de la piel moscovita; a lo que se debe adicionar que muchas de estas posiciones también esgrimían, de manera velada, la puesta en juego de intereses de todo tipo a escala regional y local que, por ejemplo, llevaron finalmente al desmantelamiento de la Escuela Normal Superior ${ }^{8}$.

Las tensiones en un escenario marcado por una coyuntura tan radical y con la incertidumbre en el horizonte no permiten valorar los esfuerzos para alentar la adquisición y la producción del conocimiento científico que se implementó en este mismo periodo; es decir, muchas de las medidas gubernamentales hacen evidente las preocupaciones por estudiar a la sociedad colombiana y la necesidad de crear mecanismos para difundir el conocimiento a un nivel amplio dentro de los diferentes sectores sociales, al mismo tiempo, que permitieran una comprensión más detallada de la realidad nacional mediante la creación de instituciones para formar una nueva intelectualidad. Por eso, es necesario prestar atención a la política cultural del periodo.

$\mathrm{Al}$ aceptar esta perspectiva como punto de partida de análisis, la Revista de las Indias deja de ser un medio de propaganda del partido liberal colombiano para extenderse como una apuesta a favor de una concepción del Estado nacional. La nueva función de la revista se pone en escena en un momento de crisis mundial que impuso una política cultural dirigida a crear mecanismos de difusión que permitieran el acceso a la mayor cantidad de personas, de los productos culturales elaborados por los escritores, los artistas plásticos y los hombres de letras colombianos y extranjeros ${ }^{9}$.

\section{Política cultural para la unidad nacional: la extensión}

En el periodo que va entre 1880 y 1930, Colombia vivió importantes transformaciones políticas, económicas y sociales que consolidaron al Estado nacional en medio de

\footnotetext{
${ }^{7}$ Villegas, Silvio. No hay enemigos a la derecha (Manizales: Editorial Zapata, 1937); Alzate Avendaño, Gilberto. "El redescubrimiento del Libertador", La Patria, Manizales, 30 de julio de 1938.

${ }^{8}$ Reyes, Aura Lisette. "Educando al educador, el caso de la Escuela Normal Superior", en Baukara. Bitácoras de Antropología e Historia de la Antropología en América Latina, núm. 1, Bogotá, Universidad Nacional de Colombia, 2012, pp. 34-54; Herrera, Martha Cecilia. Modernización y Escuela Nueva en Colombia, 1914-1951 (Bogotá: Universidad Pedagógica Nacional, 1999); Herrera Cortés, Martha Cecilia y Low P., Carlos. El caso de la Escuela Normal Superior. Los intelectuales y el despertar cultural del siglo: una historia reciente y olvidada (Bogotá: Universidad Pedagógica Nacional, 1994).

${ }^{9}$ Estoy de acuerdo con la apreciación de Renán Silva en el siguiente aspecto: la mayoría de los estudios sobre este periodo, incluso hasta trabajos muy recientes, insisten en el valor político partidista de las reformas generadas bajo los gobiernos del partido liberal, y cuando asumen el plano cultural el énfasis del análisis se dirige a la política educativa, una esfera vital en estas reformas pero que no abarca el proceso general, la formulación de una política cultural de parte del Estado nacional en donde la educación era solo uno de sus componentes. Silva Olarte, Renán. "Política cultural e inmigración docente en el marco de la República Liberal”, en Historia y Sociedad, núm. 24, Medellín, Universidad Nacional de Colombia, 2013, pp.19-51.
} 
una extraordinaria inestabilidad institucional: tres guerras civiles: 1885,1895 y la Guerra de los Mil Días (1899-1902), la pérdida de Panamá (1903) y el quinquenio autoritario de Rafael Reyes (1904-1909). Estos procesos políticos correspondían a transformaciones económicas novedosas que habían dado origen a los ciclos agroexportadores de la quina (1880), el café, el banano, la ganadería y el petróleo (1905), que dieron un importante impulso a la navegación por el río Magdalena y al despegue industrial que hacia 1930 se localizaba en Bogotá, Medellín, Barranquilla y Cali. Como correspondencia a estos procesos económicos algunas ciudades superaron los cien mil habitantes en este periodo; por ejemplo, Bogotá tenía en 1870 aproximadamente 40.000 habitantes y hacia 1938 tenía 330.000 habitantes; Medellín pasó en el mismo periodo de 30.000 a 168.000 habitantes; Cali de 13.000 a 102.000 habitantes; sin embargo, la mayoría de las cabeceras municipales oscilaban entre 5.000 y 20.000 habitantes, a lo que debe añadirse el papel crucial de la Iglesia católica en el ámbito de la educación, a pesar de sus limitaciones en la estructura educativa y las bajas tasas de escolaridad. El papel central de la Iglesia católica llegó hasta el punto que muchos gobernantes de este periodo asimilaron las tareas del Estado con los fines de la Iglesia. En esta transición de siglos “[...] la vida cultural colombiana se desenvolvió en medio de las tensiones provocadas por el dominio ejercido por el catolicismo" $" 10$ y los intentos por secularizar esta situación a través de embates modernizadores que se pueden observar en la publicación de trabajos como Idola Fori (1909), de Carlos Arturo Torres; La vorágine (1924), de José Eustasio Rivera; la extensa y reconocida obra de José María Vargas Vila (1860-1933); la apertura del Teatro Colón en Bogotá (1892); la fundación del colegio Gimnasio Moderno (1914) y la Universidad Libre (1923); entre otras iniciativas que marcaron una transformación de la cultura letrada colombiana ${ }^{11}$.

Entre los años 1930 y 1940, Colombia vivió una serie de iniciativas relacionadas con modificaciones constitucionales para impulsar la apertura al reconocimiento y la aplicación de derechos sociales. También vivió las vicisitudes para respaldar la creación de algunas instituciones que pudieran abarcar procesos de alcance nacional moderno y democrático en el campo de la educación, la cuestión agraria y la industrialización ${ }^{12}$. En este contexto, la política cultural debía abarcar todo el espectro de la sociedad y debía aplicarse como una acción única de la nación, el departamento y el municipio para enfrentar:

[...] el desorden que la guerra ha traído consigo en el ámbito espiritual y en la órbita de la técnica, la política, la economía y la sociología, en general. Con mira a evitar futuras sorpresas, que podrían quebrantar nuestras instituciones

\footnotetext{
${ }^{10}$ Londoño, Patricia. "La cultura", en Posada Carbó, Eduardo (Dir.), Colombia: la apertura al mundo (1880-1939) (Madrid: Fundación Mapfre-Taurus, 2015); Escovar Wilson-White, Alberto. "Bogotá en tiempos de la celebración del primer Centenario de la Independencia”, en Historia Mexicana, vol. LX, núm. 1, México, El Colegio de México, 2010, pp. 525-559.

${ }^{11}$ Ibid.

${ }^{12}$ Silva, Renán. "Libros, lecturas y lectores durante la República Liberal”, en Silva, Renán, República liberal, intelectuales y cultura popular (Medellín: La Carreta Editores, 2005), pp. 87-185.
} 
democráticas, conviene, desde ahora, realizar una cruzada en favor de las adquisiciones de la cultura por medio de los organismos estatales ${ }^{13}$.

El gobierno enfrentaba altas tasas de analfabetismo de la población adulta; de allí que era necesario atacar el problema de la escuela para que no siguiera ampliándose el universo de analfabetas adultos, y extender los productos del conocimiento científico y letrado entre las sociedades campesinas para elevar sus niveles de vida, de lectura $\mathrm{y}$, sobre todo, para integrarlas a la comunidad nacional al "[...] facilitar al campesino colombiano la mayor suma posible, dentro de las condiciones actuales de la República, de bienestar material y de dignidad espiritual, para que ame la vida que le cupo en suerte, y la sirva con efectiva estimación y gratitud"14.

Para llegar a la sociedad adulta que no había participado de la precaria institucionalidad educativa y cuyo referente letrado estaba relacionado con la convivencia cercana de la vida eclesial, era necesario que el gobierno apelara a los recursos técnicos disponibles: el cine, la radio y las actividades de extensión cultural. Por eso, la política cultural entre 1930 y 1946 se trazó, inicialmente, bajo la forma del proyecto de Cultura Aldeana concebido por el Ministro de Educación, Luis López de Mesa (1934-1935), durante el gobierno de Alfonso López Pumarejo (1934-1938).

La base de aquella propuesta era la definición de la aldea colombiana entendida como el municipio o corregimiento que tuviera entre 500 a 5.000 habitantes y tuviera un poblado como centro administrativo. El proyecto debía ser ejecutado por una Comisión de Cultura Aldeana compuesta por expertos en urbanismo y salubridad pública, en agronomía pedagogía escolar y un relator literario, perito en sociología. La Comisión, afirmaba López de Mesa, debía recorrer las aldeas y enseñar a sus habitantes lo que deben hacer para mejorar su nivel de vida, al mismo tiempo, que debía determinar en el terreno, las reformas necesarias para mejorar la prosperidad económica, la estética de la personalidad y del ambiente, la cultura de la mente y la disciplina del carácter; además, debía coordinar las actividades de las Comisiones filiales en cada Departamento ${ }^{15}$.

En este contexto de diagnóstico y de la vulgarización científica, las bibliotecas jugaron un papel central. El proyecto de las Bibliotecas Aldeanas y las colecciones de libros

\footnotetext{
${ }^{13}$ Achury, Darío. "Extensión Cultural", en Ministerio de Educación Nacional, La Extensión Cultural en 1944 (Bogotá: Prensas de la Biblioteca Nacional, 1944), pp. 12-13.

${ }^{14}$ López de Mesa, Luis. "Estatuto de la Aldea Colombiana", en Senderos. Biblioteca Nacional de Colombia, vol. II, núms. 7 y 8, Bogotá Biblioteca Nacional de Colombia, 1934, p. 2.

${ }^{15}$ La Comisión con apoyo del Ministerio de Educación podía elaborar planos para casas de habitación de acuerdo a las condiciones climáticas de cada lugar; sugerir la construcción de parques con jardines, piscinas y avenidas para pasear y para promover que los aldeanos practicaran deportes; apoyar la construcción de una Casa Social como salón para festividades; instalar un cinematógrafo y aparatos de radiodifusión; implementar la biblioteca aldeana; promover una misión docente para construir la escuela aldeana; verificar y gestionar la presencia de un médico, y donde fuera necesario, proveer el puesto de abogado de pobres. López de Mesa, Luis. "Estatuto de la Aldea Colombiana", en Senderos. Biblioteca Nacional de Colombia, vol. II, núms. 7 y 8, Bogotá Biblioteca Nacional de Colombia, 1934, pp. 2-5.
} 
que las integrarían se bosquejaron y realizaron bajo la política de Cultura Aldeana del gobierno de López Pumarejo. Esta política permitió la creación de bibliotecas municipales, las llamadas Bibliotecas Aldeanas, que estaban constituidas por una serie de colecciones de libros que fueron trazadas por Luis López de Mesa y Daniel Samper Ortega de la siguiente forma:

I. Colección Cartillas Técnicas, nacionales y extranjeras.

II. Colección Información para la Segunda Enseñanza o Conocimientos Generales, que debían ser textos para el estudio introductorio de las ciencias, así como manuales de oficios prácticos que fueron integradas por libros de la Casa Appleton Century Company y la editorial Seix Barral.

III. Colección Literatura Universal o de Obras de Entretención, compuesta por la Colección Araluce complementada con una serie de biografías de grandes hombres y la Colección Fabre sobre el mundo animal.

IV. Colección Selección Samper Ortega de literatura colombiana también conocida como Biblioteca Aldeana de Colombia, integrada por cien obras editadas por la Editorial Minerva, que se constituyó paulatinamente en la primera colección que se hizo del pensamiento colombiano ${ }^{16}$.

En 1936, el Ministerio de Educación realizó un censo sobre esta labor y reportó la existencia de 674 Bibliotecas Aldeanas en todo el país, a las que habían llegado 95.462 ejemplares impresos y contabilizó 125.890 usuarios de dichas bibliotecas ${ }^{17}$.

\section{La Biblioteca Nacional y sus tareas de difusión}

La política de Cultura Aldeana del gobierno de Alfonso López Pumarejo tuvo en la biblioteca uno de sus principales ejes de acción porque ella no solo era un repositorio de libros para consulta, también se convirtió en un centro de difusión de actividades culturales -conferencias, proyección de películas, espacio para escuchar música- y, sobre todo, un lugar para la enseñanza de la lectura. La biblioteca, entonces, debía ser un "organismo de difusión de la cultura, y la cultura de la aldea"18. El modelo de todas estas acciones recayó en las funciones atribuidas a la Biblioteca Nacional en el marco de esta política cultural; tales fines fueron trazados bajo la orientación de Daniel Samper Ortega, director de la Biblioteca Nacional entre 1931 y 1938.

Samper Ortega estableció que la biblioteca ya no podía ser entendida como un depósito de libros sino como un organismo donde el libro es escasamente un elemento más de

\footnotetext{
${ }^{16}$ Silva señala que estas colecciones tuvieron que integrarse con materiales extranjeros adquiridos casi por azar debido a la dificultad para encontrar libros similares en la escasa producción editorial nacional. Mientras tanto, la selección Samper fue objeto de ataques desde la política partidista que señalaban su carácter academicista, excluyente y a favor del gobierno. Silva, Renán. "Libros, lecturas y lectores durante la República Liberal”, en Silva, Renán. República liberal, intelectuales y cultura popular (Medellín: La Carreta Editores, 2005), pp. 90-104.

${ }^{17}$ Muñoz Vélez, Hernán Alonso. La Biblioteca Aldeana de Colombia y el ideario de la República Liberal. Bibliotecas y cultura en Antioquia, 1934-1947 (Bogotá: Universidad del Rosario, 2013); Herrera, Martha Cecilia y Jilmar Díaz, Carlos. "Bibliotecas y lectores en el siglo XX colombiano: la Biblioteca Aldeana de Colombia”, en Revista Educación y Pedagogía, vol. XIII, núms. 29-30, Medellín, Universidad de Antioquia, 2001, pp. 103-111.

${ }^{18}$ Ibid.
} 
culturización; es decir, las bibliotecas tenían que servir de centros de irradiación de cultura por todos los medios posibles. El proyecto de Samper Ortega consideraba que la biblioteca debía tener las siguientes secciones:

I. Librería: para establecer una red de bibliotecas y llenar el país de bibliotecas.

II. Editorial: para recolectar e imprimir el pensamiento colombiano y extranjero con una producción anual entre 100 y 200 libros para distribuirse en la red de bibliotecas porque era preciso movilizar las ideas, utilizarlas como medicina para curarle al país la anemia intelectual que lo mata.

III. Radio: para superar el analfabetismo de los campesinos, poder brindar consejo y mantener la verdadera unidad nacional.

IV. Cinematografía: como el medio más efectivo de enseñanza y como medio de difusión de cualquier ministerio.

Para complementar estas funciones, Samper Ortega precisaba que el carácter nacional de la Biblioteca Nacional se daría siempre y cuando hubiera sucursales -las Bibliotecas Aldeanas- en las capitales de los departamentos y en las ciudades con más de 20.000 habitantes para que la Biblioteca Nacional no fuera solo una institución de la capital ${ }^{19}$.

En el diseño de Samper Ortega los trabajos de la Biblioteca no podían recaer solo en los libros. Las acciones para difundir la cultura y el conocimiento implicaban trascender la presencia sustancial de los libros. Cada uno de esos planteamientos tuvo la atención y la gestión de Samper Ortega como director, quien concretó el trabajo de radiodifusión a partir de la instalación de antenas y equipos en el Capitolio Nacional, dando origen en 1932 a la emisora H. J. N., la cual inició labores en 1940 y paulatinamente, se convirtió en el antecedente de la Radiodifusora Nacional de Colombia ${ }^{20}$.

Dentro de esta prolija actividad de difusión, la Biblioteca Nacional diseñó y patrocinó la publicación de la revista Senderos como el órgano oficial de la Biblioteca y como una revista concebida para promover el patriotismo y el arte, asuntos de los que no se había ocupado el Estado nacional hasta ese momento; de tal manera que Senderos debía ser un medio para superar las dificultades que enfrentaron las iniciativas privadas para la producción y difusión de la literatura y el arte colombiano. Hasta ese momento, la iniciativa privada fue la estrategia para elaborar, difundir y preservar al quehacer letrado que estructuró las tradiciones literarias colombianas desde mediados del siglo

\footnotetext{
${ }^{19}$ Para cumplir con estas tareas, Samper Ortega presentó un proyecto al Ministerio de Educación para adquirir la imprenta, la radiodifusora con los equipos receptores y cincuenta equipos ambulantes de cinematografía. Samper Ortega, Daniel. “Sobre los propósitos del Ministerio de Educación”, en Senderos. Biblioteca Nacional de Colombia, vol. II, núms.7 y 8, Bogotá, Biblioteca Nacional de Colombia, 1934, pp. 6-11.

${ }^{20}$ Samper Ortega, Daniel, "Informe del Director de la Biblioteca Nacional al Señor Ministro de Educación”, en Senderos. Biblioteca Nacional de Colombia, vol. I, núm. 5, Bogotá, Biblioteca Nacional de Colombia, 1934, pp. 215-222; Silva, Renán. "Ondas nacionales. A propósito de la creación de la Radiodifusora Nacional de Colombia”, en Silva, Renán, República liberal, intelectuales y cultura popular (Medellín: La Carreta Editores, 2005), pp. 59-86. La propia Biblioteca Nacional pudo tener un edificio diseñado explícitamente para sus funciones cuando se inauguró el 20 de julio de 1938.
} 
XIX, pero sus avatares demostraban que estos proyectos editoriales habían llegado a ser esfuerzos importantes en su labor, pero empresas efímeras en su duración ${ }^{21}$; por eso, el propósito de Senderos era:

[...] mejorar la facha y de meollo para llevar a los pueblos hermanos la vibración de Colombia, de una Colombia nueva, que está resuelta a recuperar su alto prestigio intelectual en el continente; y al propio tiempo aspira a ser entre nosotros el hilo que cosa unas con otras las diversas regiones de esta tierra, difundiendo en todas ellas mutuos anhelos y necesidades ${ }^{22}$.

La revista Senderos bajo la dirección de Daniel Samper Ortega no pudo superar los antecedentes de las iniciativas dedicadas a la difusión de la cultura letrada en Colombia. En diciembre de 1935 dio aviso sobre el fin de esta empresa por instrucción directa del Ministerio de Educación Nacional, a pesar de que la revista había alcanzado 23 números reunidos en 4 volúmenes publicados entre febrero de 1934 y diciembre de 1935.

\section{La Revista de las Indias: un paso más en la extensión cultural}

Las pretensiones de la difusión hacia la que propendía la política cultural del gobierno de López Pumarejo adquirieron un nuevo matiz con la llegada del gobierno de Eduardo Santos. Desde 1938 se creó la Dirección de Extensión Cultural del Ministerio de Educación Nacional con el objetivo de irradiar la cultura a la sociedad colombiana $^{23}$, y para ello se estructuraron paulatinamente una serie de departamentos administrativos relacionados con estas labores: Museos y Exposiciones; Radiodifusora Nacional de Colombia; Teatro Colón; Teatro Cultural; Orquesta Sinfónica Nacional; Banda Nacional; Escuelas y Conservatorios de Música Departamentales; Escuela Complementaria de Especialización Artística y Centro de Cultura Social -dibujo, canto y danza-; Educación Física; Instituto Rufino J. Cuervo -a partir de 1944, Instituto Caro y Cuervo-; Servicio de Arqueología -Instituto Etnológico Nacional-; y el de Cultura Popular y Publicaciones.

La organización de la Dirección de Extensión Cultural supone dos aspectos de la cultura; el primero de ellos referido a la cultura popular, que se refería básicamente a la intención del Estado de "[...] dar los pasos iniciales para dotar al pueblo de elementos primarios que vayan conformando su vida estética [...] la educación estética: la música, la poesía, la pintura, como ejercicio creador o como simple ejercicio contemplativo, realizan profundas transformaciones en el alma humana"24.

\footnotetext{
${ }^{21}$ Loaiza Cano, Gilberto. Manuel Ancizary suépoca (1811-1882). Biografía de un político hispanoamericano del siglo XIX (Medellín: Editorial Universidad de Antioquia- Fondo Editorial Universidad EAFIT, 2004).

22 Daniel Samper Ortega. "Editorial”, en Senderos. Biblioteca Nacional de Colombia, vol. I, núm. 1, Bogotá, Biblioteca Nacional de Colombia, 1934, p. 1.

23 “Decreto Número Núm. 1965 de 1938 (3 de noviembre de 1938), Orgánico del Ministerio de Educación Nacional", Diario Oficial, Bogotá, 15 de noviembre de 1938.

${ }^{24}$ Achury, Darío. "Cultura Popular", en Ministerio de Educación Nacional, La Extensión Cultural en 1945 (Bogotá: Prensas de la Biblioteca Nacional, 1945), p. 45.
} 
Por lo tanto, esta parte del ministerio tenía que ver con la organización de campañas de difusión como los conciertos populares, la proyección de películas educativas, las ferias del libro, las obras de teatro, las conferencias culturales diversas, las escuelas ambulantes, los concursos de arte, entre otras actividades.

Al mismo tiempo, la Dirección de Extensión tuvo un segundo aspecto relacionado con el fomento de otro aspecto de la cultura:

[...] todos aquellos valores que no tienen cotización y realización inmediatamente útil para el ejercicio comicial, han venido a ser desplazados y considerados por el Estado como cosa sobrante, inútil y aplazable. Y comoquiera que el vivir puramente espiritual, el fomento del arte, la investigación científica, se refieren a objetos en cierto modo intemporales, ocurre que la pura cultura no tiene carta de naturaleza, ni ambiente adecuado, ni campo propicio dentro de la enorme órbita que ocupa en la sociedad el Estado $^{25}$.

Por eso, la Dirección de Extensión Cultural partía de la intención del Estado de ir a la inteligencia y de solicitar el esfuerzo de los hombres de egregia naturaleza, para que se dedicaran fundamentalmente a la investigación científica y el trabajo especializado en el arte, la literatura, la filosofía, la investigación histórica y filológica; es decir, el Estado tenía que impulsar el trabajo de instituciones dedicadas a los estudios superiores como el Instituto Caro y Cuervo, el Instituto Etnológico Nacional, la Comisión Nacional de Folklore y publicaciones como la Revista de la Academia de Ciencias Exactas, Físicas y Naturales, y la Revista de las Indias.

Dentro de la organización de la Dirección de Extensión Cultural los servicios adscritos a la Sección de Cultura Popular incluían las publicaciones, y dentro de estas labores estaban por igual las revistas especializadas que se han mencionado al mismo tiempo que la principal empresa de difusión de la producción intelectual colombiana emprendida por el Ministerio de Educación Nacional en el periodo entre 1930-1946: la Biblioteca Popular de Cultura Colombiana, que fue una colección de libros que se publicó desde 1942 en el marco de una estrategia para recopilar y dar a conocer la historia y la literatura colombiana a partir del antecedente que representó la Selección Samper Ortega de Literatura Colombiana, como parte de las acciones emprendidas a través de la organización y la realización de las Ferias del $\mathrm{Libro}^{26}$.

\footnotetext{
${ }^{25}$ Rocha, Antonio. "Introducción”, en Ministerio de Educación Nacional, La Extensión Cultural en 1944 (Bogotá: Prensas de la Biblioteca Nacional, 1944), p. 11.

${ }^{26}$ Catálogo de la Biblioteca Popular de Cultura Colombiana y de otros volúmenes editados por la Sección de Publicaciones del Ministerio de Educación Nacional (Bogotá: Prensas del Ministerio de Educación Nacional, 1948). Las diferencias entre las iniciativas de difusión de las tradiciones letradas colombianas que implican las colecciones Samper Ortega y la Biblioteca Popular en Silva, Renán. "Las Ferias del Libro en Colombia, 1936-1947”, en Silva, Renán, República liberal, intelectuales y cultura popular (Medellín: La Carreta Editores, 2005), pp. 187-221. No se puede perder de vista que en este periodo también circularon publicaciones como la Revista del Instituto Etnológico Nacional (1942-1950) y El Boletín de Arqueología (1947) que no aparecen en los informes citados.
} 
La Revista de las Indias en sus inicios hizo parte de la política de extensión cultural de los gobiernos colombianos entre 1936 y 1938, ya que era financiada y publicada por el Ministerio de Educación dentro de la esfera de la promoción de la alta cultura, pero en 1938 esta tuvo un giro particular a raíz de dos elementos circunstanciales, sobre los que no se va a ahondar en este trabajo. El primero de ellos tiene que ver con las iniciativas impulsadas por la Unión Americana para fomentar las actividades de Cooperación Intelectual, acciones en las que participaron diferentes integrantes de la revista como Baldomero Sanín Cano y Germán Arciniegas como puede establecerse en la presencia de al menos uno de ellos como delegado de Colombia en la Octava Conferencia Internacional Americana de 1938, en el Primer Congreso Americano de Comisiones Nacionales de Cooperación Intelectual de 1939, y en la Segunda Conferencia de Comisiones Nacionales de Cooperación Intelectual de 1941.

El otro acontecimiento fue un evento cultural que se desarrolló en el marco de los festejos del Cuarto Centenario de la fundación de Bogotá. Entre el 4 y el 14 de septiembre de 1938 se organizó una exposición y una feria de libros cuyo acto central fue la reunión para constituir la Sociedad de Escritores Americanos y Españoles, sociedad que acordó publicar una revista como órgano de difusión ${ }^{27}$.

En este contexto, el Ministerio de Educación del gobierno colombiano convino que la Revista de las Indias fuera el órgano de difusión de aquella asociación y suscribió un contrato que estableció que la revista sería una publicación del Ministerio de Educación Nacional con fines de divulgación cultural e información literaria y que estaría bajo la dirección y administración de la Sociedad de Autores Americanos y Españoles. Estas labores serían ejercidas por la asociación sin remuneración alguna

El contrato señalaba que los empleados requeridos serían sufragados con el producto de la propia publicación, a excepción de una mecanógrafa pagada por el ministerio; también obligaba a la asociación a publicar mensualmente un ejemplar de la revista y a ejecutar todas las acciones administrativas y legales que tuvieran que ver con la integración, producción y distribución de cada número. No obstante, el contrato permitía que la revista manejara los dineros recaudados por concepto de avisos, suscripciones, venta de ejemplares y suplementos, previa autorización de las tarifas por el ministerio, para la publicación de obras de carácter literario, científico o artístico dentro del plan de divulgación cultural de la asociación.

Así mismo, el contrato señaló que la asociación debía entregar gratuitamente a la Biblioteca Nacional trescientos ejemplares de la revista y cien ejemplares de los suplementos que llegara a publicar, al igual que las publicaciones que recibiera como parte del canje. En este tenor, el contrato obligaba al ministerio a designar una partida presupuestal anual correspondiente a los costos de edición de cada número

\footnotetext{
27 "Resolución 186 por la cual se concede Personería Jurídica a la Asociación de Escritores Americanos y Españoles del 26 de septiembre de 1938”, Diario Oficial, Bogotá, 4 de febrero de 1939. Esta Resolución certificaba el domicilio de la asociación en Bogotá y como Presidente de la misma, así como representante legal, a Germán Arciniegas.
} 
(papel, litografía, fotograbados, carátulas, etc.), a brindar una oficina equipada para la asociación; a conceder libre porte de los correos nacionales para la correspondencia y ejemplares de la revista y sus suplementos, y a mantener la vigencia del contrato por tres años ${ }^{28}$.

La revista contaba con un auxilio del gobierno colombiano, pero la mayor parte del financiamiento de la publicación, entre 1938 y 1943, se mantuvo a través de la gestión de la publicidad, las suscripciones y los canjes que se convirtieron en los ingresos para hacer el pago del administrador, el correo internacional y, sobre todo, de las colaboraciones; así que no era una publicación que funcionara a expensas, completamente, del gobierno colombiano. También, estaba claramente estipulado que la Dirección y la gestión de los contenidos de la publicación recaían en la asociación, lo cual colocaba a los funcionarios del Ministerio de Educación en una posición fiscalizadora básicamente en los temas referidos a la contabilidad y el ejercicio de los recursos, lo cual no dejó de generar una serie de tensiones entre las personas que realizaban las gestiones de ambas partes en diferentes momentos ${ }^{29}$.

La particular situación administrativa de la revista permitió la continuidad editorial y temática de la publicación cuando se observa su desenvolvimiento en el periodo durante el cual apareció y que abarcó los años de 1936 a 1950. Este aspecto es crucial si se contrasta con la percepción que se ha mantenido hasta ahora de que esta hacía parte de una entidad pública nacional como medio de propaganda y donde la característica es la discontinuidad de los funcionarios; lo cual se puede desmentir cuando se advierte que durante su vida editorial tuvo los siguientes directores:

I. Arcadio Dulcey, primer director que dirigió los volúmenes I y II; es decir, desde el número 1 de julio de 1936 hasta el número 10 de agosto de 1938.

II. German Arciniegas, que inició la segunda época, lo que implicó una nueva numeración. Arciniegas dirigió desde el número 1 de enero de 1939 hasta el número 91 de julio de 1946.

\footnotetext{
28 "Contrato entre el Gobierno y la Sociedad de Autores sobre la dirección y administración de la Revista de Indias, 20 de febrero de 1939" y "Memorandum del Contrato de la "Revista de las Indias" para el Señor Ministro de Educación, 18 de marzo de 1939”, Biblioteca Nacional de Colombia (BNC), Fondo Germán y Gabriela Arciniegas, c. 22, car. 14; "Decreto 319 del 13 de febrero de 1939 por el cual se organiza la dirección y administración de la Revista de las Indias", Diario Oficial, febrero 13 de 1939. El contrato fue firmado por Alfonso Araujo, como Ministro de Educación del gobierno de Colombia, y Germán Arciniegas, a la sazón, representante legal de la Asociación ante el Ministerio. El contrato también preveía que la Revista colocara en la carátula una leyenda "Revista de las Indias publicada por el Ministerio de Educación de Colombia, y a cargo de la Asociación de Escritores Americanos y Españoles”, aviso que se mantuvo en la carátula hasta el año de 1943.

${ }^{29}$ Los informes y las cartas de los administradores de la revista como Camilo Restrepo, José Lloreda Camacho, Alberto Miramón, y algunos otros documentos del archivo de Germán Arciniegas revelan las diferentes tensiones y dificultades que planteaban estas situaciones administrativas y dejaban en claro los periodos de precariedad y abundancia que dependían de circunstancias como, por ejemplo, si Arciniegas "sonaba" para ser Ministro; al mismo tiempo, dan cuenta de los problemas que tenían los responsables de la revista para publicar los números mensualmente, aspecto que cumplieron en contadas ocasiones. En estos mismos documentos no hay evidencia de intervención del ministerio en los contenidos de la revista. A partir del mes de mayo de 1944, la revista y los empleados al servicio de esta pasaron a depender directamente del Ministerio de Educación.
} 
III. Cuando fue nombrado Ministro de Educación en el periodo 1945-1946, Arciniegas nombró a Jorge Rojas como director de Extensión Cultural en reemplazó de Darío Achury Valenzuela; en este movimiento, Jaime Posada fue nombrado redactor, cargo que ejerció desde el número 92 de agosto de 1946 hasta el número 95 de enero de 1947.

IV. Abel Naranjo Villegas fue el director desde el número 96 de mayo de 1947 hasta el número 101 de enero-marzo de 1948.

V. José María Vivas Balcázar, director desde el número 102 de abril-mayo de 1948 hasta el número 109 de mayo-junio de 1949.

VI. Jaime Vélez Sáenz, desde el número 110 de julio-septiembre de 1949 hasta el número 115 de septiembre-octubre de 1950.

La Revista de las Indias se organizó en diferentes secciones que se ajustaron con el tiempo, pero mantuvieron una tendencia de continuidad en lo general: Artículos diversos; Sección temática; Poesía; Notas (noticias sobre diversos asuntos de la vida nacional e internacional, así como novedades sobre letras, plásticas, ciencias); Libros; y en diversos momentos, especialmente hacia el final de la existencia de la publicación aparecieron secciones como: El mes internacional; Geografía literaria de Colombia y suplementos diversos sobre teatro y música, cuentos, poemas. Los textos se publicaban a solicitud de los editores y sus corresponsales se ubicaban en todo el continente. Este aspecto organizacional es un hecho novedoso ya que complementa la idea de una reflexión sobre lo nacional a partir de autores locales para dar cuenta del mundo desde Colombia. Una experiencia similar no se había dado hasta ese momento, excepto la interesante empresa que representó la revista Hispania (19121916) materializada en Londres por la iniciativa de Santiago Pérez Triana, Enrique Pérez Lleras y Baldomero Sanín Cano, como un milagro intelectual en medio de las vicisitudes de la Primera Guerra Mundial ${ }^{30}$.

La revista organizó una extensa red de colaboradores internacionales que eran reconocidos en su campo; por ejemplo, en el número 68 de agosto de 1944, Germán Arciniegas fungía como director y el cuerpo de la Redacción lo integraban: Baldomero Sanín Cano (Colombia), Luis de Zulueta (España), Herschel Brickell (Estados Unidos), Gustavo Adolfo Otero (Bolivia), Gonzalo Zaldumbide (Ecuador) y Juan Guzmán Cruchaga (Chile), que permitió integrar números con textos de Pablo Neruda (Chile), Paul Valéry (Francia), Jorge Guillén (Cuba), Gabriela Mistral (Chile), Rafael Heliodoro Valle (Honduras), Miguel Otero Silva (Venezuela), Francisco Ayala (España), Alfred Coester (Estados Unidos), Ezequiel A. Chávez (México), Gerhard Masur (Alemania), Natalicio González (Paraguay), entre otros muchos autores de reconocido prestigio internacional, que daban lustre a la revista y enmarcaban los aportes de los principales autores colombianos del momento: Baldomero Sanín Cano, Eduardo Carranza, Eduardo Caballero Calderón, Otto de Greiff, Rafael Maya, Luis Vidales, Jorge Zalamea, Luis Eduardo Nieto Arteta, José Antonio Osorio Lizarazo, Germán Pardo García, Guillermo Hernández de Alba.

\footnotetext{
${ }^{30}$ Castaño Duque, Gildardo. "Revista Hispania (1912-1916): presencia cultural colombiana en la vida intelectual europea", en Vivas Hurtado, Selnich (coord.), Utopías móviles. Nuevos caminos para la historia intelectual en América Latina (Bogotá: Diente de León Editor-Universidad de Antioquia, 2014), pp. 32-63.
} 
En la parte de los contenidos, la revista también incluyó múltiples espectros temáticos: filosofía, historia, ciencia natural, derecho, economía, poesía, crítica literaria acorde con el esfuerzo sobre la apertura hacia diferente tipo de colaboradores, para lo cual utilizó los mecanismos disponibles para ello: pagó por los textos, traducciones, reseñas informativas, organización de algunos números temáticos, usó las notas explicativas para escritos que presentaban temas novedosos, especialmente del ámbito científico, y, sobre todo, mantuvo las corresponsalías extranjeras.

Si bien existía la apertura temática y el planteamiento de tener una mirada más allá de las fronteras nacionales, la revista no abandonó la coyuntura interna. Aunque, la temática nacional no era el marco de referencia para la integración de los diferentes números, los editores no eran ajenos a su entorno inmediato y presentaban opiniones acerca de los acontecimientos nacionales. Por ejemplo, una situación tan impactante como los acontecimientos del 9 de abril de 1948 llevó a los editores a publicar un escrito en el que, además de lamentar la muerte de Jorge Eliécer Gaitán, deploraban los días nefandos que siguieron a su muerte; de tal suerte que hicieron pública la siguiente postura, a pesar de la militancia de la revista en el partido político de Gaitán:

A nombre de la cultura nacional, de la cual esta Revista es vocero y tribuna, dejamos constancia de nuestra protesta contra quienes desde las radiodifusoras lanzaron al pueblo enfurecido, primero contra el gobierno legítimamente constituido y contra la ciudad entera, después.

Amargo resulta relatar, siquiera en síntesis, las pérdidas de valores artísticos y culturales sufridas irreparablemente [...] No sobra recordar que en el solo Palacio Arzobispal fueron destruidos quince mil volúmenes de su valiosa biblioteca. [...] ¿A nombre de qué podemos defender a quienes tales estragos promovieron, encauzaron y consumaron? Los que tal catástrofe engendraron no son simples delincuentes contra el presente, lo son contra el pasado y contra el porvenir. ¡Las manos de nuestros próceres los acusan y las de nuestros hijos también los maldicen! ${ }^{31}$.

\section{Reiteración de una forma de hacer: la escritura ensayística}

A pesar de los enroques burocráticos de la figura de Germán Arciniegas en la década de 1940 que lo pusieron al frente de la Revista de las Indias al mismo tiempo que desempeñaba labores como ministro, representaciones diplomáticas y como profesor invitado en instituciones universitarias en los Estados Unidos, hizo explícitas algunas de sus consideraciones sobre la línea editorial y los fines de la publicación:

[...] una Revista fundamentalmente literaria. Su público es un público de elite [...] En realidad yo no he estado de acuerdo nunca con la idea de que el Ministerio de Educación, que es una entidad puramente administrativa, dirija por conducto de uno de sus empleados publicaciones literarias de ningún género. No tiene el Ministerio por qué entrar a seleccionar material literario [...] Yo preferiría

31 "Nuestra protesta", en Revista de las Indias, núms. 102-103, Bogotá, Ministerio de Educación Nacional, 1948, pp. 511-513. 
buscarle a la Revista una dirección autónoma perfectamente responsable e interesada en hacer de la Revista de las Indias la mejor publicación literaria de su género en el país, y si fuera posible, en América. Tengo la ambición de hacerlo ${ }^{32}$.

Estas declaraciones dan precisión a un hecho central, cuando se habla de la revista como un órgano de la cultura implica en ello que la cultura corresponde al ámbito letrado; es decir, en esta se privilegió básicamente los asuntos relacionados con las humanidades pero con un énfasis particular en la literatura a la que sus editores consideraban como el medio idóneo para dar cuenta de la realidad americana y servir como vehículo de afirmación de la identidad del continente, de sus relaciones profundas con las herencias culturales europeas, al mismo tiempo que reafirmaba la independencia cultural frente a España y a toda Europa; por lo tanto, la literatura era concebida como un documento social y la mejor forma de expresar las especulaciones del espíritu y de representar a la realidad; además, la literatura era un medio que tenía la posibilidad de identificarse fácilmente con los lectores ${ }^{33}$.

La valoración de la literatura, un tema ampliamente discutido dentro de las mismas tradiciones literarias en América Latina, tiene que ver, especialmente, con la disponibilidad de conocimiento; es decir, en un momento donde no existía la profesionalización de los saberes y apenas se daban los primeros pasos en la institucionalización de muchos de ellos, las representaciones para comprender y explicar la realidad social y los procesos históricos adquirieron fundamentalmente las variadas formas de la literatura: la poesía, el teatro, la novela, el cuento y el ensayo. La literatura se asimiló como el producto más visible e inmediato de la actividad intelectual, a pesar de no ser una profesión, porque el mundo letrado en América Latina asumió como su principal función social ser la punta de lanza de una gran empresa formativa porque "La literatura es la más perceptible expresión de la complejidad histórica de un pueblo, la que le da conciencia de lo que es, cómo ha llegado a ser y lo que quiere llegar a ser"34. La literatura, en cualquiera de sus géneros, se equiparó a la alta cultura, sus cultivadores en la concreción del espíritu letrado, y sus productos, la mejor fuente de autoridad para opinar sobre cualquier asunto. Por eso, en el momento que existió la revista para comprender la realidad bastaba ser un hombre de letras para estar en capacidad de explicar el presente y el pasado. ${ }^{35}$

\footnotetext{
32 “Cambios en la Revista de Indias”, $B N C$, Fondo Germán y Gabriela Arciniegas, c. 8, car. 6.

${ }_{33}$ Marín Colorado, Paula Andrea. "Revista de las Indias (1936-1951): vehículo de expresión de una conciencia americana”, en Alma Mater. Agenda Cultural de la Universidad de Antioquia, núm. 201, Medellín, Universidad de Antioquia, 2013, pp. 16-18.

${ }^{34}$ Gutiérrez Girardot, Rafael. "Conferencia del 20-XI-87”, en Gutiérrez Girardot, Rafael, Temas y problemas de una historia social de la literatura hispanoamericana (Bogotá: Cave Canem, 1989), p. 97.

${ }^{35}$ Gaos, José. "El pensamiento hispano-americano. Notas para una interpretación histórico-filosófica", en Pensamiento de Lengua Española. Obras Completas, vol. VI (México: Universidad Nacional Autónoma de México, 1990), pp. 30-107; Gutiérrez Girardot, Rafael. "La literatura colombiana en el siglo XX", en Jaramillo Uribe, Jaime (Dir.), Manual de Historia de Colombia, t. III (Bogotá: Instituto Colombiano de Cultura, 1980), pp. 445-536.
} 
Las consideraciones sobre la alta cultura y el papel de los hombres de letras en la sociedad fue una preocupación latente en toda América Latina en estos momentos de crisis. En el caso colombiano, esta preocupación se manifiesta, por ejemplo, en la encuesta que preparó y difundió Estampa: revista semanal de actualidad gráfica, entre febrero y marzo de 1939, que se concentró en dos preguntas: “¿Cuál debe ser la actitud de los intelectuales ante el dilema: Fascismo-democracia?, ¿cuál es el autor que más ha influido en su formación intelectual y por qué razones?" "36. Las preguntas tuvieron las más variadas respuestas que demuestran las opciones disponibles en un momento en el que las consideraciones sobre el presente eran bastantes inciertas ${ }^{37}$. Mientras transcurría la Segunda Guerra Mundial, el periódico El Espectador, entre marzo y abril de 1942, convocó a otra encuesta alrededor de tres preguntas: “¿Cuál es su posición ante los dos campos ideológicos en que está hoy dividido el mundo, y cuáles las razones en que ella se funda?, ¿qué resultados e influencias tendrá la organización mundial que ha salir de la guerra sobre el arte a que está usted dedicado?, ¿cuál cree usted que deba ser la organización moral, política y económica de este mundo de postguerra?" "38; sin embargo, esta iniciativa, en comparación con la que le antecedió, tuvo poca acogida ${ }^{39}$.

El desenvolvimiento de la Segunda Guerra Mundial era seguido con preocupación por la cultura letrada, y, sobre todo, causaba intranquilidad el destino de Europa como modelo de cultura. Una muestra de ello se encuentra en la encuesta a los intelectuales que presentó la Revista de las Indias en el número 72 de diciembre de 1944 y que publicó sucesivamente hasta el número 77, en mayo de 1945, estructurada en cuatro preguntas:

1. ¿Permanecerán en Europa o cambiarán de sede los centros de nuestra cultura en la posguerra?

2. ¿Seguirá a los actuales trastornos un progreso o una decadencia en la marcha de la cultura?

3. ¿En el porvenir se les asignará valor de medios o de fines a la literatura y al arte?

\footnotetext{
36 "Los intelectuales ante el fascismo y ante la democracia", en Estampa: revista semanal de actualidad gráfica, vol. VIII, Bogotá, 1939. La encuesta la respondieron: Rafael Maya, Luis Eduardo Nieto Caballero, Luis Vidales, Eduardo Carranza, Germán Arciniegas, Juan Francisco Mujica, Rodrigo Jiménez Mejía, León de Greiff, Esteban Jaramillo, Antonio García, Carlos H. Pareja, Carlos Vesga Duarte, Jorge Eliecer Gaitán, Alberto Miramón, Luis Eduardo Nieto Arteta, Parmenio Cárdenas, Antonio Gómez Restrepo, Rafael Carrillo, Jorge Soto del Parral, Luis Felipe Latorre y Alfonso Fuenmayor. La extensión de cada respuesta, producto de una entrevista, impide realizar un análisis en este trabajo.

${ }^{37}$ Ibid.

38 "Nuestra encuesta", en El Espectador, 23 de febrero de 1942. La encuesta tuvo menos acogida y las respuestas fueron menos extensas y más espaciadas en el tiempo. Esta encuesta la respondieron: Darío Achury, Carlos Restrepo Piedrahita, Antonio J. Lemus, Baldomero Sanín Cano, Manuel Serrano Gómez, Jaime Paredes, Pedro Gómez Parra, Jaime Ardila Casamitjanas y Manuel de J. Romero.

${ }^{39}$ Ibid.
} 
4. ¿Qué papel reserva el futuro a los intelectuales y, especialmente, a escritores y artistas? ${ }^{40}$.

La solicitud y las respuestas las conseguía la revista de manera escrita; aun así, las respuestas obtenidas fueron muy desiguales en atención y profundidad; destaca, por ejemplo, que Sanín Cano se tomó ocho cuartillas para responder, mientras Francisco Ayala presentó dos cuartillas. Al final, las respuestas publicadas fueron de: Rafael Maya, Baldomero Sanín Cano, J. Rodríguez Paramo, Francisco Ayala, Jorge Bayona Posada, Cayetano Betancur, Ramón Gómez de la Serna, Guillermo Díaz Doin, Jaime Tello Quijano, Mariano Ruiz-Funes, Luis Vidales, José Prat, Pedro Salinas, J. M. Restrepo Millán y Francisco José González, S. J.

Las preguntas y las respuestas de aquella encuesta coincidían en un aspecto: concebían a Europa como la madre de la cultura occidental y a la literatura como una expresión trascendente del espíritu humano, que no iba a desaparecer ni a cambiar en su finalidad última. A pesar de que muchas respuestas eran genéricas, es evidente la preocupación por el futuro y las condiciones para la producción intelectual de la posguerra. Aunque hay incertidumbre por el futuro de Europa como referente, una gran parte de los encuestados mantiene la confianza en que Europa seguirá siendo el centro cultural que había sido:

Me resisto a creer que la construcción ingente de la cultura europea, en su rango hoy primario, se venga abajo con la facilidad con que se han derrumbado las fábricas arquitectónicas materiales bajo el azote de los bombardeos. Ciertas voces agoreras, prolongando con sus ecos las profecías, dan a Europa por difunta, o agonizante, y se aprestan a enterrarla con más o menos honores [...] la Europa de hoy $[\ldots]$ sigue siendo lo que fue ${ }^{41}$.

El tono optimista de Salinas contrasta con las consideraciones de Baldomero Sanín Cano que al valorar la situación hacía notar que:

[...] la brújula de la inteligencia comienza a dar indicios de graves perturbaciones. Es difícil pronosticar en estas materias; pero no es temerario decir que hay anuncios de cambio. Uno de ellos es el interés que despierta el estudio de la lengua inglesa, otro es la efervescente actividad, la tenacidad del pueblo ruso en el desarrollo de sus nuevas generaciones, otro la vitalidad literaria de los Estados Unidos.

\footnotetext{
40 "Notas. Encuesta a los Intelectuales", en Revista de las Indias, núm. 72, Bogotá, Ministerio de Educación Nacional, 1944, pp. 439-449; "Encuesta a los Intelectuales", Revista de las Indias, núm. 73, Bogotá, Ministerio de Educación Nacional, 1945, pp. 118-126; "Encuesta a los Intelectuales”, Revista de las Indias, núm. 74, Bogotá, Ministerio de Educación Nacional, 1945, pp. 271-282; "Encuesta a los Intelectuales", Revista de las Indias, núm. 75, Bogotá, Ministerio de Educación Nacional, 1945, pp. 417-426; "Reflexiones sobre la cultura. A propósito de la Encuesta a los Intelectuales”, Revista de las Indias, vol. LXXVI, Bogotá, Ministerio de Educación Nacional, 1945, pp. 6-18 y "Encuesta a los Intelectuales", Revista de las Indias, núm. 77, Bogotá, Ministerio de Educación Nacional, 1945, pp. 288-295.

${ }^{41}$ Salinas, Pedro. "Reflexiones sobre la cultura. A propósito de la Encuesta a los Intelectuales", en Revista de las Indias, vol. LXXVI, Bogotá, Ministerio de Educación Nacional, 1945, pp. 6-7.
} 
Acaso la facilidad de comunicaciones, la difusión de la prensa diaria y periódica, la ubicuidad de la radio, hagan imposible hoy la fijeza de un meridiano literario como el que solíamos reconocer hace sesenta años ${ }^{42}$.

Las observaciones sobre el desplazamiento del meridiano cultural que hizo Sanín Cano ponían sobre la mesa el papel que debía cumplir América en este escenario de incertidumbre.

\section{A modo de cierre abierto: América Latina, un tema pendiente desde Colombia}

La descripción más amplia del proyecto y el desenvolvimiento de la Revista de las Indias se debe al trabajo de Manuel Restrepo, que hizo parte de esa interesante iniciativa del Boletín Cultural y Bibliográfico del Banco de la República para estudiar las revistas culturales colombianas. El trabajo de Restrepo destaca el esfuerzo de la revista por ir más allá de las fronteras nacionales con base en la estrategia de abordar y mostrar a América Latina en sus diferentes facetas: poesía, ficción, crítica literaria; sin embargo, se queja Restrepo porque:

Lamentablemente, la otra cara de la moneda mostró que un equipo tan calificado como el que tuvo la dirección de la revista no logró crear conciencia sobre esa 'gran utopía intelectual' que tiene como mira la preocupación por la expresión cultural latinoamericana [...] Estos discursos no alcanzaron eco en las comunidades literarias y científicas, pero tampoco lograron conformar un proyecto sustitutivo [...] Lo lamentable del asunto fue que, frente a esa innegable y vigorosa tarea de organizar una extensa red de colaboradores internacionales de gran calidad, muy escasos fueron los frutos que se recogieron, en el sentido de dar respuesta a las grandes preguntas sobre el destino de este continente, o al menos de crear corrientes sobre ellas ${ }^{43}$.

Las consideraciones de Restrepo sobre la Revista de las indias merecen atención en ciertos aspectos. El primero de ellos, es la constatación de que la revista demuestra los vínculos internacionales de algunos integrantes del cuerpo editorial de la publicación como Arciniegas y Sanín Cano; lo cual fue un buen punto de partida para establecer el sello de la revista y convertirla en una tarea que no se había realizado hasta entonces en Colombia; además, el enfoque multitemático, atento a la realidad inmediata, a la situación internacional y al acontecer del mundo letrado se convirtió en un modelo, en muchos sentidos, para publicaciones como Mito: Revista Bimestral de Cultura (1955-1962), ya que las principales figuras de esta legendaria empresa cultural que colaboraron en la parte final de la revista, aunque con desigual presencia: Jorge Gaitán Durán, Eduardo Cote Lamus, Eduardo Zalamea Borda y Hernando Tellez.

\footnotetext{
42 "Notas. Encuesta a los Intelectuales", en Revista de las Indias, vol. LXXII Bogotá, Ministerio de Educación Nacional, 1944, p. 444.

${ }^{43}$ Restrepo, Manuel. "Revista de las Indias, un proyecto de ampliación de fronteras", en Boletín Cultural y Bibliográfico, vol. XXVII, núm. 23, Bogotá, Banco de la República, 1990, p. 26.
} 
El segundo aspecto que se resalta es el tema del desencanto que tiene Restrepo sobre las respuestas que podía ofrecer la revista sobre el destino del Continente y la conciencia sobre esa gran utopía intelectual. En este rubro, es necesario señalar que Colombia durante el siglo XX tuvo, y mantiene aún, una política que valora de manera positiva la alineación con las directrices de los Estados Unidos. En este periodo, el gobierno colombiano buscó articularse, de manera consciente, a los principios del panamericanismo y a la defensa del bloque del liberalismo económico y la democracia como proyecto político. Este énfasis se presenta en la revista y se manifiesta de una manera explícita en la participación de Germán Arciniegas, como director, en el evento organizado por la Comisión Cubana de Cooperación Intelectual en La Habana, donde se planteó la defensa de las tesis panamericanistas de la libertad, la democracia y la justicia social. En este aspecto, el cuerpo editorial de la revista fue consecuente con los lineamientos del gobierno colombiano y con la División de Cooperación Intelectual de la Unión Panamericana que tuvo en la Asociación de Escritores y Artistas Americanos uno de sus principales baluartes. La revista, entonces, tomó partido y eso no implica que ella hubiera tenido un "especial desencuentro con las situaciones que ha vivido el continente", como dice Restrepo.

Al igual que las lecturas desde la política partidista, la mirada sobre América Latina tiene puntos de partida y suposiciones sobre cuál es la postura políticamente correcta. Creo que los integrantes de la revista la asumieron y consideraron que ella atendía esa preocupación, solo que esto ocurrió dentro de la perspectiva del panamericanismo y no de otro proyecto político. ¿Eso la hace menos latinoamericanista? Es un punto que necesita ser desarrollado con más atención y por eso, sigue como una tarea pendiente en el espectro académico colombiano.

\section{Bibliografía}

\section{Fuentes Primarias}

Biblioteca Nacional de Colombia (BNC), Fondo Germán y Gabriela Arciniegas.

Catálogo de la Biblioteca Popular de Cultura Colombiana y de otros volúmenes editados por la Sección de Publicaciones del Ministerio de Educación Nacional. Bogotá: Prensa del Ministerio de Educación Nacional, 1948.

\section{Prensa}

Diario Oficial, Bogotá, 1939.

La Patria, Manizales, 1938.

Revista de las Indias, 1936-1950.

Senderos. Biblioteca Nacional de Colombia, 1934. 


\section{Artículos de prensa}

Álzate Avendaño, Gilberto. "El redescubrimiento del Libertador", en La Patria, julio 30 de 1938.

\section{Fuentes Secundarias}

\section{Libros}

Ayala Diago, César Augusto. El porvenir del pasado. Gilberto Álzate Avendaño: sensibilidad leoparda y democracia. La derecha colombiana de los años treinta. Bogotá: Fundación Gilberto Álzate Avendaño-Universidad Nacional de Colombia, 2007.

Ayala Diago, César Augusto. Inventando al Mariscal: Gilberto Álzate Avendaño: circularidad ideológica y mímesis política. Bogotá: Fundación Gilberto Álzate Avendaño-Universidad Nacional de Colombia, 2010.

Herrera Cortés, Martha Cecilia. Modernización y Escuela Nueva en Colombia, 19141951. Bogotá: Universidad Pedagógica Nacional, 1999.

Herrera Cortés, Martha Cecilia y Low P., Carlos. El caso de la Escuela Normal Superior. Los intelectuales y el despertar cultural del siglo: una historia reciente y olvidada. Bogotá: Universidad Pedagógica Nacional, 1994.

Loaiza Cano, Gilberto. Manuel Ancizar y su época (1811-1882). Biografía de un politico hispanoamericano del siglo XIX. Medellín: Editorial Universidad de Antioquia-Fondo Editorial Universidad EAFIT, 2004.

Martínez Hernández, Lina María. La Revista de las Indias (1936-1938): sus intelectuales como pensadores y ejecutores de la reforma educativa y cultural. Bogotá: Universidad de los Andes, 2011.

Ministerio de Educación Nacional. La obra educativa del Gobierno en 1940: la extensión cultural en Colombia, t. 3. Bogotá: Imprenta Nacional, 1940.

Morales Benítez, Otto (comp.). Baldomero Sanín Cano: ideología y cultura, t. 6. Bogotá: Universidad Externado de Colombia, 1998.

Muñoz Vélez, Hernán Alonso. La Biblioteca Aldeana de Colombia y el ideario de la República Liberal. Bibliotecas y cultura en Antioquia, 1934-1947. Bogotá: Universidad del Rosario, 2013.

Tirado Mejía, Álvaro. Aspectos políticos del primer gobierno de Alfonso López Pumarejo: 1934-1938. Bogotá: Instituto Colombiano de Cultura, 1981.

Villegas, Silvio. No hay enemigos a la derecha. Manizales: Editorial Zapata, 1937. 


\section{Capítulos de libros}

Achury, Darío. "Extensión Cultural”, en La Extensión Cultural en 1944. Bogotá: Prensas de la Biblioteca Nacional, 1944.

Achury, Darío. “Cultura Popular”, en La Extensión Cultural en 1945. Bogotá: Prensas de la Biblioteca Nacional, 1945.

Castaño Duque, Gildardo. "Revista Hispania (1912-1916): presencia cultural colombiana en la vida intelectual europea", en Vivas Hurtado, Selnich (coord.), Utopías móviles. Nuevos caminos para la historia intelectual en América Latina. Bogotá: Diente de León Editor-Universidad de Antioquia, 2014.

Gaos, José. "El pensamiento hispano-americano. Notas para una interpretación histórico-filosófica", en Gaos, José, Pensamiento de Lengua Española. Obras Completas, vol. VI. México: Universidad Nacional Autónoma de México, 1990.

Gutiérrez Girardot, Rafael, "La literatura colombiana en el siglo XX", en Jaramillo Uribe, Jaime (Dir.), Manual de Historia de Colombia, t. III. Bogotá: Instituto Colombiano de Cultura, 1980.

Gutiérrez Girardot, Rafael. "Conferencia del 20-XI-87", en Gutiérrez Girardot, Rafael, Temas y problemas de una historia social de la literatura hispanoamericana. Bogotá: Cave Canem, 1989.

Londoño, Patricia. "La cultura", en Posada Carbó, Eduardo (Dir.), Colombia: la apertura al mundo (1880-1939). Madrid: Fundación Mapfre-Taurus, 2015.

Rocha, Antonio. “Introducción”, en Ministerio de Educación Nacional, La Extensión Cultural en 1944. Bogotá: Prensas de la Biblioteca Nacional, 1944.

Sierra Mejía, Rubén. "Política y cultura durante la República Liberal”, en Sierra Mejía, Rubén (ed.), República Liberal: sociedad y cultura. Bogotá: Universidad Nacional de Colombia, 2009.

Silva, Renán. "Libros, lecturas y lectores durante la República Liberal”, en Silva, Renán, República liberal, intelectuales y cultura popular. Medellín: La Carreta Editores, 2005.

Silva, Renán. "Ondas nacionales. A propósito de la creación de la Radiodifusora Nacional de Colombia”, en Silva, Renán, República liberal, intelectuales y cultura popular. Medellín: La Carreta Editores, 2005.

\section{Artículos en revista}

Escovar Wilson-White, Alberto. "Bogotá en tiempos de la celebración del primer Centenario de la Independencia”, en Historia Mexicana, vol. LX, núm. 1, 2010. 
Herrera, Martha Cecilia y Jilmar Díaz, Carlos. "Bibliotecas y lectores en el siglo XX colombiano: la Biblioteca Aldeana de Colombia”, en Revista Educación y Pedagogía, vol. XIII, núms. 29-30, 2001.

Marín Colorado, Paula Andrea. "Revista de las Indias (1936-1951: vehículo de expresión de una conciencia americana", en Alma Mater. Agenda Cultural de la Universidad de Antioquia, núm. 201, 2013.

López de Mesa, Luis. "Estatuto de la Aldea Colombiana", en Senderos. Biblioteca Nacional de Colombia, vol. II, núms. 7 y 8, 1934.

Restrepo, Manuel. "Revista de las Indias, un proyecto de ampliación de fronteras", en Boletín Cultural y Bibliográfico, vol. XXVII, núm. 23, 1990.

Reyes, Aura Lisette. "Educando al educador, el caso de la Escuela Normal Superior", en Baukara. Bitácoras de Antropología e Historia de la Antropología en América Latina, núm. 1, 2012.

Salinas, Pedro. "Reflexiones sobre la cultura. A propósito de la Encuesta a los Intelectuales", en Revista de las Indias, núm. 76, 1945.

Samper Ortega, Daniel. "Informe del Director de la Biblioteca Nacional al Señor Ministro de Educación”, en Senderos. Biblioteca Nacional de Colombia, vol. I, núm. $5,1934$.

Samper Ortega, Daniel. "Sobre los propósitos del Ministerio de Educación", en Senderos. Biblioteca Nacional de Colombia, vol. II, núms. 7 y 8, 1934.

Silva Olarte, Renán. "Política cultural e inmigración docente en el marco de la República Liberal”, en Historia y Sociedad, núm. 24, 2013.

\section{Tesis}

Acevedo Gaviria, Claudia Patricia. Intelectuales, críticos y modernidad cultural. Los casos de Baldomero Sanín Cano, Hernando Téllez y Jorge Zalamea, (tesis doctorado), Universidad Nacional de Colombia, 2013. 